\title{
Racial Disparities in Survival Outcomes of Prostate Cancer Patients after Surgery for Bone Metastases
}

\author{
Robert L. Satcher ${ }^{1}$, Oluwatosin Bamidele ${ }^{2}$, Patrick Lin ${ }^{1}$, Sue-Hwa Lin ${ }^{3}$, Bryan Moon ${ }^{1}$, \\ Mike Hernandez ${ }^{4}$, Valerae Lewis ${ }^{1}$, Janice Chilton ${ }^{4}$
}

${ }^{1}$ Department of Orthopedic Oncology, The University of Texas MD Anderson Cancer Center, Houston, USA; ${ }^{2}$ School of Public Health, University of Texas Health Science Center, Houston, USA; ${ }^{3}$ Department of Translational Molecular Pathology, The University of Texas MD Anderson Cancer Center, Houston, USA; ${ }^{4}$ Department of Health Disparities Research, The University of Texas MD Anderson Cancer Center, Houston, USA.

Email: rlsatcher@mdanderson.org,tosinbams@gmail.com,slin@mdanderson.org,jchilton@mdanderson.org

Received February $27^{\text {th }}, 2013$; revised March $29^{\text {th }}, 2013$; accepted April $6^{\text {th }}, 2013$

Copyright (C) 2013 Robert L. Satcher et al. This is an open access article distributed under the Creative Commons Attribution License, which permits unrestricted use, distribution, and reproduction in any medium, provided the original work is properly cited.

\begin{abstract}
Introduction: This study reviewed patients' demographic, clinical and treatment characteristics to identify prognostic factors associated with survival of prostate cancer after developing bone metastases. We explored the racial disparities in these factors and how they relate with survival. Methods: We conducted a retrospective study on 79 men diagnosed with bone metastasis secondary to prostate cancer who underwent surgery at a single institution from November 1977 to June 2011. Descriptive statistics were used to summarize patients' characteristics. The Kaplan-Meier method was used to estimate characteristics of the survival distribution using two origination points - diagnosis and surgery. Cox hazard regression explored the relationship between prognostic factors and overall survival. Results: The majority of men were White $(\mathrm{n}=63 ; 80 \%)$ followed by Black $(\mathrm{n}=7 ; 9 \%)$, Hispanic $(\mathrm{n}=7 ; 9 \%)$, and Asian $(\mathrm{n}=2 ; 2 \%)$. Multivariate factors associated with poorer survival after bone metastasis surgery included race (Black), Gleason score $>8$, and radiation treatment. Patients not receiving radiation had a longer survival experience relative to patients who received radiation before or after surgery $(10.3$ vs 6.5 months; $P=0.030)$. There was an association of PSA level at the time of bone metastasis diagnosis with survival following diagnosis but prior to surgery. The median time interval $\left(\mathrm{T}_{\mathrm{m}}\right.$ in months) between prostate cancer diagnosis and bone metastasis diagnosis was 39.1 (White), 31.2 (Hispanic), 15 (Blacks) and 43 (Asians). Patients with $\mathrm{T}_{\mathrm{m}}<35$ months had a hazard of death 3 times greater than patients with $\mathrm{T}_{\mathrm{m}}>35$ months $(\mathrm{HR}=$ $3.22 ; P<0.001)$. Conclusion: The median survival and time interval from prostate cancer diagnosis to bone metastasis diagnosis was shorter in Blacks with respect to other races. The more aggressive nature of the disease in Blacks is likely due to the biology of the disease rather than access to treatment.
\end{abstract}

Keywords: Metastasis; Bone; Prostate Cancer; Racial; Gleason Score

\section{Introduction}

Patients with prostate cancer metastatic to bone have a poor prognosis; numerous studies demonstrate that survival depends on the extent of metastasis to the skeleton at presentation [1-5]. Recent studies have noted that African American men have a higher prostate cancer incidence and mortality rate than do white men [6-10]. Researchers have hypothesized that these differences are the product of both socioeconomic status [11-13], which often leads to a later diagnosis of advanced-stage prostate cancer in African American men than in white men, and of poorly understood genetic differences [11,12].

More recent evidence has clarified the role of socio- economic status in these poor outcomes among AfricanAmerican men with prostate cancer metastatic to bone. Tangen et al. reported that current trends in the survival rates of African American men with prostate cancer no longer differ from the survival rates of other men with a comparable diagnosis [14]. These investigators hypothesized that the basis of this improvement is greater awareness of prostate cancer and improved health-seeking behavior among African American men. Prostate Specific Antigen screening which began around 1990 accounted for a large proportion of these changes. This was encouraged in all men irrespective of their socioeconomic status. However, African American men 40 - 
69 years of age continue to have 2 to 3 times the incidence of newly diagnosed metastatic prostate cancer than white men of the same age have, even after adjusting for socioeconomic status [5]. The reasons for the greater incidence of advanced disease among African American men remain unknown.

Evidence supporting a biological basis for racial disparities in prostate cancer disease progression has been recently reported. Castro et al. reported that genomic profiling of comparable cohorts of African American and white men with prostate cancer showed that clinically localized cancers from African American men more closely resembled metastatic lesions from white men [6]. Wallace et al. reported prominent differences in tumor immunobiology between African American and white men with prostate cancer, with a 2-gene signature that differentiated African American and white patients [10]. These investigators found that metastasis-promoting genes were also more highly expressed in African American patients. In addition, Brand et al. reported a polymorphism in the transforming growth factor beta 1 gene that has a more protective effect against high-grade prostate cancer in non-Hispanic white men than in other racial groups [15]. In this study, we looked for further evidence of disease outcomes that depend on race, and possibly biological differences, in patients undergoing surgery for bone metastases.

The formation of skeletal metastasis negatively affects overall survival in patients with prostate cancer, and surgical intervention is considered to be palliative in the majority of these cases [16]. Surgery is meant to alleviate skeletal complications, such as bone pain, impaired mobility, pathological fracture, spinal cord compression and symptomatic hypercalcemia $[17,18]$. However, whether there is racial difference in survival time after development of bone metastasis is unknown. To investigate potential association between survival and race, we retrospectively reviewed the data for survival rates of men with prostate cancer after they had developed bone metastases requiring surgical intervention. All of the data were collected in one single institution. We first identified prognostic factors associated with survival and further examined whether race had a significant role.

\section{Methodology}

This retrospective medical chart review used data from men with prostate cancer seen at The University of Texas MD Anderson Cancer Center in Houston from November 1977 to June 2011. The research protocol was approved by the institutional review board, and a research assistant worked on chart selection and data extraction. Data were double-checked by a research coordinator to ensure accurate data collection and documentation. We searched the MD Anderson Orthopedic Oncology data base specifically for patients referred to the department as a result of bone metastasis secondary to prostate cancer requiring surgery. Inclusion criteria are men who were diagnosed with bone metastasis secondary to prostate cancer (confirmed by tissue biopsy), who fall within the 34 years range of study and who required surgical intervention for pathologic fracture, impending pathologic fracture, or intractable pain. There was no exclusion criterion. A total number of 79 men were identified for review. Their medical charts were reviewed from first visit to last follow-up or death. Data on the demographics, dates of diagnosis of prostate cancer and bone metastasis, PSA level, Gleason score, dates of discharge or death and treatment modes were extracted from their clinical records. The dates of death of subjects lost to follow-up were extracted from the genealogy bank. Data on ethnicity was self-reported and classified into African American, White, Hispanic, or Asian. Surgery was categorized according to the mode of tumor excision, and androgen-deprivation therapy was classified by leuprolide acetate (Lupron) use.

\subsection{Bone Metastasis Secondary to Prostate Cancer}

Most charts $(\mathrm{n}=76)$ included the date bone metastasis was diagnosed. Metastasis was confirmed by x-ray prior to surgery and by tissue biopsy afterward. The majority of these 76 individuals presented with bone metastasis after being diagnosed with prostate cancer $(67 ; 88 \%)$. The remaining 12\% (9) of them were diagnosed with prostate cancer and bone metastases on the same day. For the current analysis, we measured the median time intervals in months between prostate cancer and bone metastasis diagnosis and compared them among the 4 racial groups.

Gleason score and the prostatic-specific antigen (PSA) level at the time of diagnosis of prostate cancer are used to assess tumor burden [19-24].

\subsection{Data Analysis}

Data were analyzed using STATA (version 12). Descriptive statistics were used to summarize patient, clinical, and treatment-related characteristics. The Kaplan and Meier method was used to estimate characteristics of the survival distribution using 2 origination points - diagnosis and surgery [25]. The median overall survival at 1,3 , 5 and 10 years from both diagnosis and surgery were computed for the entire study population and by clinical factors of interest (i.e, age, race, Gleason grade, and type of surgery). In the analysis of overall survival, death from any cause was the event of interest, and patients' data were censored at their date of last follow-up. Cox 
proportional hazards regression was then used to explore the relationship between potential prognostic factors and overall survival at $1,3,5$ and 10 years.

\section{Results}

Seventy nine (79) individuals were identified who underwent surgery for prostate cancer bone metastases between 1977 and 2011. Baseline demographics for the study group are summarized in Table 1. The mean age at diagnosis of prostate cancer was 62 years (range: 41 - 83). The majority of men were white $(\mathrm{n}=63 ; 80 \%)$, followed by African Americans $(n=7 ; 9 \%)$, Hispanics $(n=7 ; 9 \%)$, and Asians $(n=2,3 \%)$. Of the 54 men with a Gleason score recorded at diagnosis, $37 \%$ were in the highest category $(9-10), 48 \%$ were mid-range $(7-8)$, and $15 \%$ were low $(\leq 6)$. Metastatic deposits in bone were excised from $57(72 \%)$ men. Of these patients, 67\% (38) had a wide resection, and 33\% (19) had curettage. Of the 79 patients, 44 received no radiation $(56 \%), 20$ (25\%) received post-surgery radiation only, and the remaining 5 $(6 \%)$ received pre-surgery radiation only. Only 1 patient (1\%) received radiation both before and after the surgery. The charts for 9 patients $(11 \%)$ did not report whether any radiation treatment was received.

The median survival duration following prostate cancer diagnosis in African Americans were 86\%, 28\%, and $14 \%$ at 1,3 , and 5 years, respectively $(P<0.001$, see Table 2). Survival rates after prostate cancer diagnosis were also significantly worse when the Gleason score was $9-10(P<0.001)$ and when age was younger than 65 years $(P=0.027)$. Variables that were not statistically significant were PSA level at initial diagnosis, tumor excision, type of surgery, radiation treatment, androgen-deprivation treatment, and whether bone metastases were synchronous or metachronous (Table 2). Kaplan-Meier plots of overall survival from diagnosis by race from time of survival following surgery revealed that survival was worst in African American men (Figure 1). Survival after surgery by Gleason score was worst among men with a score between 9 - 10 (Figure 2); although it did not reach statistical significance $(P=$ 0.146). However, the survival disadvantage was significant $(P=<0.001)$ from time of prostate cancer bone metastasis diagnosis (Table 2).

Following surgery for bone metastasis, median overall survival duration was 9.1 months (95\% confidence interval $[\mathrm{CI}]: 5.5,10.3$ months), with estimated survival rates at 1,3 , and 5 years of $34 \%, 10 \%$, and $9 \%$, respectively. Median survival was shortest for African American men (2.6 months, $P=0.022$; Table 3). Men who received radiation treatment either before or after surgery had less favorable in survival compared to men who did not (10.3 vs 6.5 months, $P=0.029$; Figure 3 ). There was
Table 1. Patient, clinical, and treatment characteristics.

\begin{tabular}{|c|c|}
\hline & $\mathrm{N}(\%)$ \\
\hline \multicolumn{2}{|l|}{ Age } \\
\hline Mean \pm SD & $67.1 \pm 9.6$ \\
\hline Median (range) & $68(43-89)$ \\
\hline \multicolumn{2}{|l|}{ Race or ethnicity } \\
\hline White & $63(79.7)$ \\
\hline African American & $7(8.9)$ \\
\hline Hispanic & $7(8.9)$ \\
\hline Asian & $2(2.5)$ \\
\hline \multicolumn{2}{|l|}{ PSA level at diagnosis of metastasis } \\
\hline Mean \pm SD & $187.5 \mathrm{ng} / \mathrm{mL} \pm 500.5$ \\
\hline Median (range) & $29.8 \mathrm{ng} / \mathrm{mL}(1-3004)$ \\
\hline \multicolumn{2}{|l|}{ Gleason score } \\
\hline $3-6$ & $8(14.8)$ \\
\hline $7-8$ & $26(48.2)$ \\
\hline $9-10$ & $20(37.0)$ \\
\hline \multicolumn{2}{|l|}{ Bone metastasis excised } \\
\hline No & $22(27.8)$ \\
\hline Yes & $57(72.2)$ \\
\hline \multicolumn{2}{|l|}{ Surgical category } \\
\hline Curettage & $19(24.1)$ \\
\hline No excision & $22(27.8)$ \\
\hline Wide resection & $38(48.1)$ \\
\hline \multicolumn{2}{|l|}{ Radiation } \\
\hline No radiation & $44(55.7)$ \\
\hline Pre-surgery radiation only & $5(6.3)$ \\
\hline Post-surgery radiation only & $20(25.3)$ \\
\hline Radiation both pre- and post-surgery & $1(1.3)$ \\
\hline Unknown & $9(11.4)$ \\
\hline \multicolumn{2}{|l|}{ Androgen-deprivation therapy } \\
\hline No leuprolide acetate & $18(28.1)$ \\
\hline Leuprolide acetate alone & $8(12.5)$ \\
\hline Leuprolide and other chemotherapy & $38(59.4)$ \\
\hline \multicolumn{2}{|l|}{$\begin{array}{l}\text { Bone metastasis diagnosed on same } \\
\text { day as prostate cancer }\end{array}$} \\
\hline No & $67(88.2)$ \\
\hline Yes & $9(11.8)$ \\
\hline
\end{tabular}

Abbreviations: SD, standard deviation; PSA, prostatic-specific antigen. 
Table 2. Association of patient, clinical, and treatment factors with survival after prostate cancer bone metastasis diagnosis.

\begin{tabular}{|c|c|c|c|c|c|c|c|}
\hline & \multirow{2}{*}{$\begin{array}{l}\text { Patients } \\
\text { (Deaths) }\end{array}$} & \multirow{2}{*}{$\begin{array}{c}\text { Median Survival } \\
\text { (Years) }\end{array}$} & \multicolumn{4}{|c|}{ Survival Estimates (Years) } & \multirow{2}{*}{$\begin{array}{c}\text { L-R } \\
\text { Test } \\
P\end{array}$} \\
\hline & & & 1 & 3 & 5 & 10 & \\
\hline \multicolumn{8}{|l|}{ Age } \\
\hline$\geq 65$ & $50(42)$ & 7.9 & 0.94 & 0.86 & 0.68 & 0.33 & \\
\hline \multicolumn{8}{|l|}{ Race } \\
\hline White & $63(54)$ & 6.2 & 0.97 & 0.80 & 0.64 & 0.29 & $<0.001$ \\
\hline African American & $7(7)$ & 4.9 & 0.86 & 0.28 & 0.14 & --- & \\
\hline Hispanic & $7(6)$ & 2.1 & 1.00 & 0.83 & 0.50 & 0.17 & \\
\hline Asian & $2(2)$ & --- & --- & --- & --- & --- & \\
\hline \multicolumn{8}{|l|}{ PSA level at diagnosis of metastasis } \\
\hline$<8 \mathrm{ng} / \mathrm{mL}$ & $14(11)$ & 8.7 & 1.00 & 1.00 & 0.85 & 0.31 & 0.125 \\
\hline $8-30 \mathrm{ng} / \mathrm{mL}$ & $14(13)$ & 4.6 & 0.86 & 0.71 & 0.36 & 0.07 & \\
\hline $30-100 \mathrm{ng} / \mathrm{mL}$ & $14(12)$ & 4.6 & 1.00 & 0.71 & 0.43 & 0.27 & \\
\hline$\geq 100 \mathrm{ng} / \mathrm{mL}$ & $14(12)$ & 2.4 & 1.00 & 0.47 & 0.47 & 0.28 & \\
\hline \multicolumn{8}{|l|}{ Gleason } \\
\hline $3-6$ & $8(5)$ & 10.2 & 1.00 & 1.00 & 1.00 & 0.57 & $<0.001$ \\
\hline $7-8$ & $26(21)$ & 7.9 & 1.00 & 0.84 & 0.63 & 0.28 & \\
\hline $9-10$ & $20(19)$ & 4.0 & 1.00 & 0.60 & 0.30 & 0.08 & \\
\hline \multicolumn{8}{|l|}{ Bone metastasis excised } \\
\hline No & $22(18)$ & 5.7 & 1.00 & 0.68 & 0.58 & 0.23 & 0.934 \\
\hline Yes & $57(51)$ & 5.8 & 0.95 & 0.80 & 0.60 & 0.27 & \\
\hline \multicolumn{8}{|l|}{ Surgical category } \\
\hline Curettage & $19(17)$ & 5.8 & 0.95 & 0.89 & 0.61 & 0.33 & 0.815 \\
\hline No excision & $22(18)$ & 5.7 & 1.00 & 0.68 & 0.58 & 0.23 & \\
\hline Wide resection & $38(34)$ & 5.8 & 0.94 & 0.75 & 0.59 & 0.24 & \\
\hline \multicolumn{8}{|l|}{ Radiation } \\
\hline No & $44(35)$ & 5.8 & 0.98 & 0.81 & 0.58 & 0.32 & 0.210 \\
\hline Yes $^{\mathrm{a}}$ & $26(25)$ & 5.1 & 0.96 & 0.69 & 0.58 & 0.19 & \\
\hline \multicolumn{8}{|l|}{ Androgen-deprivation therapy } \\
\hline No leuprolide acetate & $18(16)$ & 4.6 & 1.00 & 0.78 & 0.44 & 0.32 & 0.161 \\
\hline Leuprolide acetate alone & $8(4)$ & 12.6 & 1.00 & 0.68 & 0.68 & 0.51 & \\
\hline Leuprolide and other chemotherapy & $38(35)$ & 5.7 & 0.97 & 0.76 & 0.62 & 0.16 & \\
\hline \multicolumn{8}{|c|}{$\begin{array}{l}\text { Bone metastasis diagnosed on same day as prostate } \\
\text { cancer }\end{array}$} \\
\hline No & $67(60)$ & 5.8 & 0.98 & 0.80 & 0.63 & 0.25 & 0.103 \\
\hline Yes & $9(8)$ & 1.6 & 0.78 & 0.44 & 0.15 & 0.15 & \\
\hline
\end{tabular}

Abbreviations: L-R, log-rank test; PSA, prostatic-specific antigen. Insufficient data to obtain reliable estimates; ${ }^{\mathrm{a}}$ Radiation was given before, after, or both before and after surgery; we excluded data from 9 patients with unknown radiation treatment status. 


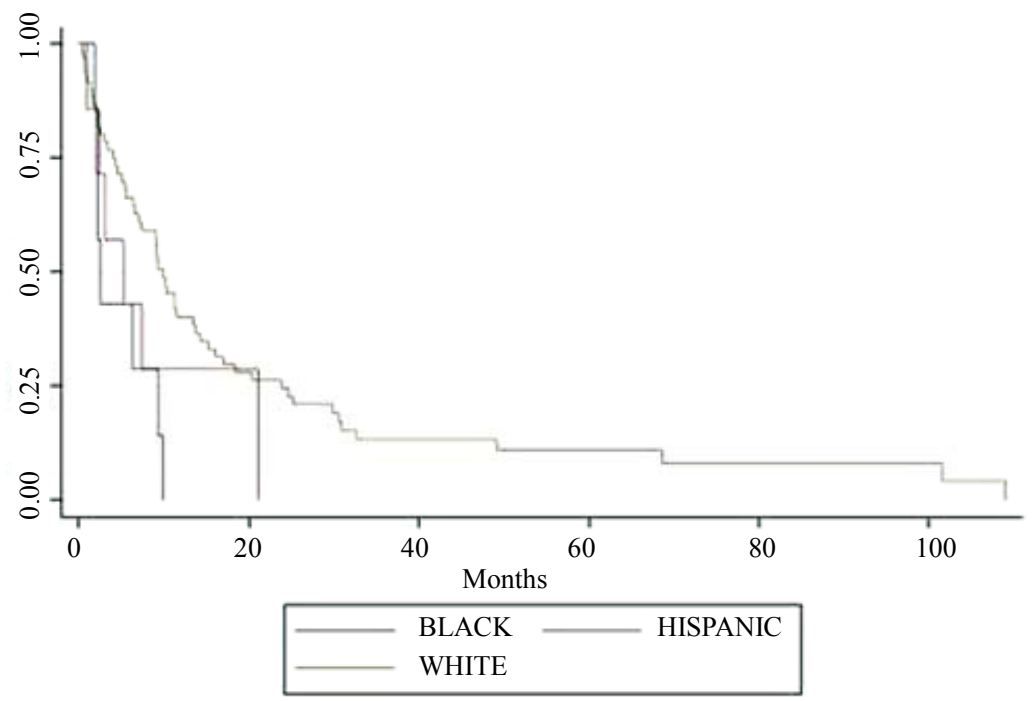

Figure 1. Kaplan-Meier plots of overall survival by race from time of surgery (months). Survival estimates in years was plotted against race. KM revealed that survival is worst in blacks as measured by steepest decline in survival estimate in relation with time in years. $P<0.05$.

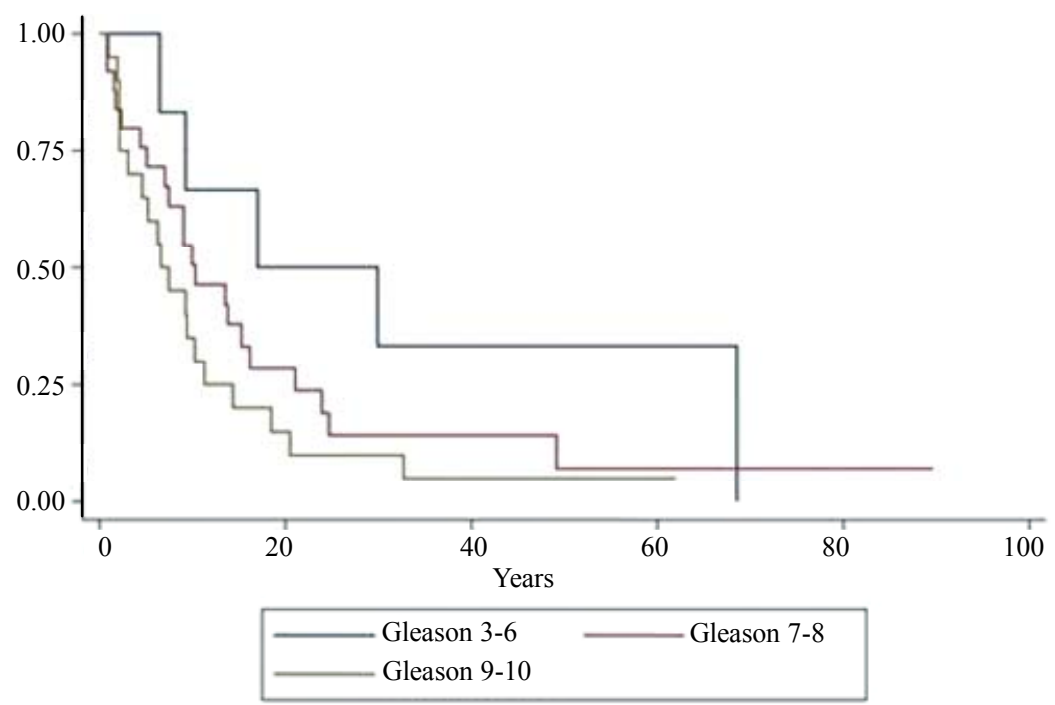

Figure 2. Kaplan-Meier plots of overall survival by Gleason score from time of surgery (months). Gleason grades 3-6 (blue line), 7-8 (red line), 9-10 (green line). Survival estimates plotted against Gleason score. KM revealed that survival is worst with Gleason scores 9-10 as measured by steepest decline in survival estimate in relation with time in years. $P>0.05$.

no statistically significant difference in survival for men with respect to age, Gleason score, tumor excision, surgical category, androgen-deprivation therapy, or having bone metastasis identified on the day of diagnosis.

Table 4 shows the hazard ratios (HR) of factors associated with survival following diagnosis and surgery. Following prostate cancer diagnosis, risk of death was highest in African American patients (HR, 5.13; P < 0.001 ) and second highest in patients with Gleason scores of 9 or 10 (HR, 5.02; $P=0.002)$. An increase in PSA level of $20 \mathrm{ng} / \mathrm{mL}$ was associated with a statistically significant but very small increase in risk (HR, 1.02; $P=$
0.002), and 5-year increments in age at diagnosis were associated with a statistically significantly lower risk (HR, $0.85 ; P=0.014$ ). Following surgery for bone metastasis, risk of death was significantly higher for African American patients (HR, 2.93; $P=0.011)$ than for other races and for patients treated with radiation (HR, 1.80; $P$ $=0.031)$. The HR for those with high Gleason scores trended toward higher risk of death following surgery but did not reach statistical significance (HR, 2.58; $P=0.065$ ).

The overall time to disease progression as determined by median time interval (months) between prostate cancer diagnosis and bone metastasis diagnosis (as shown in 
Table 3. Association of patient, clinical, and treatment factors with survival after surgery for prostate cancer bone metastatic lesions.

\begin{tabular}{|c|c|c|c|c|c|c|c|}
\hline & \multirow{2}{*}{$\begin{array}{l}\text { Patients } \\
\text { (Deaths) }\end{array}$} & \multirow{2}{*}{$\begin{array}{l}\text { Median Survival } \\
\text { (Months) }\end{array}$} & \multicolumn{4}{|c|}{ Survival Estimates (Years) } & \multirow{2}{*}{$\begin{array}{c}\text { L-R Test } \\
P\end{array}$} \\
\hline & & & .5 & 1 & 3 & 5 & \\
\hline \multicolumn{8}{|l|}{ Age } \\
\hline$<65$ & $29(27)$ & 7.4 & 0.62 & 0.31 & 0.08 & 0.08 & 0.661 \\
\hline$\geq 65$ & $50(42)$ & 9.2 & 0.61 & 0.36 & 0.13 & 0.10 & \\
\hline \multicolumn{8}{|l|}{ Race } \\
\hline White & $63(54)$ & 10.0 & 0.66 & 0.40 & 0.13 & 0.11 & 0.022 \\
\hline African American & $7(7)$ & 2.6 & 0.43 & --- & --- & --- & \\
\hline Hispanic & $7(6)$ & 5.2 & 0.43 & 0.28 & --- & --- & \\
\hline Asian & $2(2)$ & --- & --- & --- & --- & --- & \\
\hline \multicolumn{8}{|l|}{ Gleason score } \\
\hline $3-6$ & $8(5)$ & 17.0 & 1.00 & 0.67 & 0.33 & 0.33 & 0.146 \\
\hline $7-8$ & $26(21)$ & 10.3 & 0.72 & 0.46 & 0.14 & 0.07 & \\
\hline $9-10$ & $20(19)$ & 6.6 & 0.60 & 0.25 & 0.05 & 0.05 & \\
\hline \multicolumn{8}{|l|}{ Bone metastasis excised } \\
\hline No & $22(18)$ & 10.0 & 0.63 & 0.37 & 0.07 & 0.07 & 0.832 \\
\hline Yes & $57(51)$ & 9.0 & 0.61 & 0.33 & 0.12 & 0.10 & \\
\hline \multicolumn{8}{|l|}{ Surgical category } \\
\hline Curettage & $19(17)$ & 6.6 & 0.65 & 0.24 & 0.06 & 0.06 & 0.779 \\
\hline No excision & $22(18)$ & 10.0 & 0.63 & 0.37 & 0.07 & 0.07 & \\
\hline Wide resection & $38(34)$ & 9.2 & 0.60 & 0.38 & 0.15 & 0.11 & \\
\hline \multicolumn{8}{|l|}{ Radiation } \\
\hline No & $44(35)$ & 10.3 & 0.66 & 0.48 & 0.12 & 0.12 & 0.029 \\
\hline $\mathrm{Yes}^{\mathrm{a}}$ & $26(25)$ & 6.5 & 0.57 & 0.16 & 0.04 & --- & \\
\hline \multicolumn{8}{|l|}{ Androgen-deprivation therapy } \\
\hline No leuprolide acetate & $18(16)$ & 7.4 & 0.53 & 0.29 & 0.06 & 0.06 & 0.924 \\
\hline Leuprolide acetate alone & $8(4)$ & 11.3 & 0.54 & 0.27 & 0.27 & --- & \\
\hline Leuprolide and other chemotherapy & $38(35)$ & 9.3 & 0.70 & 0.40 & 0.09 & 0.06 & \\
\hline \multicolumn{8}{|c|}{ Bone Metastasis diagnosed on same day as prostate cancer } \\
\hline No & $67(60)$ & 7.4 & 0.59 & 0.32 & 0.11 & 0.09 & 0.920 \\
\hline Yes & $9(8)$ & 10.2 & 0.67 & 0.33 & --- & --- & \\
\hline
\end{tabular}

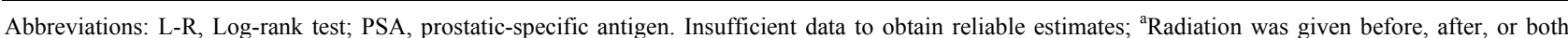
before and after surgery; we excluded data from 9 patients with unknown radiation treatment status.

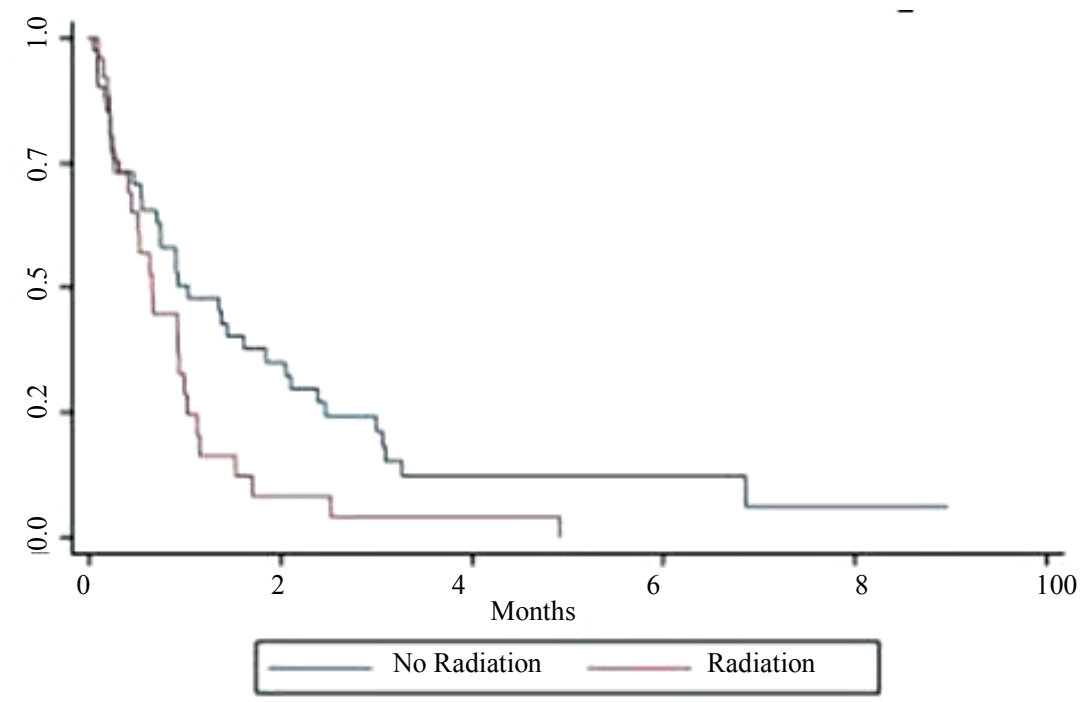

Figure 3. Kaplan-Meier plots of overall survival by radiation from time of surgery (months). Radiation + (red line); no radiation-(blue line). Survival estimates plotted against radiation. KM revealed that survival is less favorable in men that had radiation at some point as measured by a steeper decline in survival estimate in relation with time in months. $P<0.05$. 
Table 4. Factors associated with survival from prostate cancer bone metastasis diagnosis and surgery.

\begin{tabular}{|c|c|c|c|c|c|c|}
\hline \multirow{2}{*}{ Variable } & \multicolumn{3}{|c|}{ From Diagnosis } & \multicolumn{3}{|c|}{ From Surgery } \\
\hline & $P$ & HR & $95 \% \mathrm{CI}$ & $P$ & HR & $95 \% \mathrm{CI}$ \\
\hline \multicolumn{7}{|l|}{ Age in Years } \\
\hline 5 -year increments & 0.014 & 0.85 & $(0.75,0.97)$ & 0.924 & 0.99 & $(0.88,1.12)$ \\
\hline \multicolumn{7}{|l|}{ Race } \\
\hline White & \multicolumn{3}{|c|}{ Ref. } & \multicolumn{3}{|c|}{ Ref. } \\
\hline African American & $<0.001$ & 5.13 & $(2.18,12.0)$ & 0.011 & 2.93 & $(1.28,6.71)$ \\
\hline Hispanic & 0.32 & 1.54 & $(0.66,3.64)$ & 0.274 & 1.61 & $(0.68,3.80)$ \\
\hline Asian & --- & --- & --- & --- & --- & --- \\
\hline \multicolumn{7}{|c|}{ PSA level at diagnosis of metastasis } \\
\hline $20 \mathrm{ng} / \mathrm{mL}$ increments & 0.002 & 1.02 & $(1.01,1.03)$ & 0.705 & 1.00 & $(0.99,1.01)$ \\
\hline \multicolumn{7}{|l|}{ Gleason score } \\
\hline $3-6$ & \multicolumn{3}{|c|}{ Ref. } & \multicolumn{3}{|c|}{ Ref. } \\
\hline $7-8$ & 0.216 & 1.86 & $(0.70,4.96)$ & 0.242 & 1.80 & $(0.67,4.82)$ \\
\hline $9-10$ & 0.002 & 5.09 & $(1.82,14.2)$ & 0.065 & 2.58 & $(0.94,7.04)$ \\
\hline \multicolumn{7}{|l|}{ Bone metastasis excised } \\
\hline No & \multicolumn{3}{|c|}{ Ref. } & \multicolumn{3}{|c|}{ Ref. } \\
\hline Yes & 0.934 & 1.02 & $(0.60,1.76)$ & 0.832 & 1.06 & $(0.62,1.82)$ \\
\hline \multicolumn{7}{|l|}{ Surgical category } \\
\hline Curettage & \multicolumn{3}{|c|}{ Ref. } & \multicolumn{3}{|c|}{ Ref. } \\
\hline No excision & 0.774 & 1.10 & $(0.57,2.15)$ & 0.558 & 0.82 & $(0.41,1.61)$ \\
\hline Wide resection & 0.528 & 1.21 & $(0.67,2.18)$ & 0.505 & 0.82 & $(0.45,1.49)$ \\
\hline \multicolumn{7}{|l|}{ Radiation } \\
\hline No & \multicolumn{3}{|c|}{ Ref. } & \multicolumn{3}{|c|}{ Ref. } \\
\hline $\mathrm{Yes}^{\mathrm{a}}$ & 0.212 & 1.39 & $(0.83,2.33)$ & 0.031 & 1.80 & $(1.05,3.06)$ \\
\hline \multicolumn{7}{|l|}{ Androgen-deprivation therapy } \\
\hline No Leuprolide acetate & & Ref. & & & Ref. & \\
\hline Leuprolide acetate alone & 0.503 & 0.68 & $(0.22,2.09)$ & 0.727 & 0.82 & $(0.27,2.47)$ \\
\hline In combination & 0.175 & 1.55 & $0.82,2.92)$ & 0.762 & 0.91 & $(0.50,1.66)$ \\
\hline \multicolumn{7}{|c|}{ Bone metastasis diagnosed on same day as prostate cancer } \\
\hline No & & Ref. & & & Ref. & \\
\hline Yes & 0.107 & 1.89 & $(0.87,4.12)$ & 0.920 & 1.04 & $(0.49,2.19)$ \\
\hline
\end{tabular}

Abbreviations: HR, hazard ratio; CI, confidence interval; PSA, prostatic- specific antigen; Ref., reference set as a unit of 1. ${ }^{a}$ Radiation was given before, after, or both before and after surgery; we excluded data from 9 patients with unknown radiation treatment status.

Table 5) was 35 months. African American men had the shortest average time at 15 months, followed by Hispanics (31.2 months), Whites (39.1 months), and Asians (43 months). Patients with shorter time spans ( $<35$ months) from prostate cancer to bone metastasis had a hazard of death 3 times greater than that of patients with longer time spans ( $>35$ months $)(\mathrm{HR}=3.22 ; P<0.001)$. Table 6 shows the time from diagnosis of bone metastasis to surgery. The shortest median time was again for African Americans at less than a month (0.8), followed by Hispanics (2.4 months), whites (8.4 months), and Asians (58.7 months).

\section{Discussion}

Our results showed that race and radiation treatment were significantly associated with death from prostate 
Table 5. Time from date of prostate cancer diagnosis to date of bone metastasis diagnosis.

\begin{tabular}{|c|c|c|c|c|}
\hline \multirow{2}{*}{ Race } & \multirow{2}{*}{$\begin{array}{c}\text { Number of } \\
\text { Patients (76) }\end{array}$} & \multicolumn{3}{|c|}{$\begin{array}{l}\text { Interval from Diagnosis to } \\
\text { Metastasis Detection (months) }\end{array}$} \\
\hline & & Mean & Median & Range \\
\hline White & 60 & 51.8 & 39.1 & $0-276.8$ \\
\hline Hispanic & 7 & 46.6 & 31.2 & $0-128.4$ \\
\hline African American & 7 & 21.8 & 15.0 & $0-64.8$ \\
\hline Asian & 2 & 43.0 & 43.0 & $0.2-85.8$ \\
\hline
\end{tabular}

Table 6. Time from bone metastasis diagnosis to date of surgery.

\begin{tabular}{ccccc}
\hline \multirow{2}{*}{ Race } & \multirow{2}{*}{$\begin{array}{c}\text { Number of } \\
\text { Patients (71) }\end{array}$} & \multicolumn{3}{c}{$\begin{array}{c}\text { Interval from Metastasis } \\
\text { Detection to Surgery (months) }\end{array}$} \\
\cline { 3 - 5 } & & Mean & Median & Range \\
\hline White & 55 & 17.6 & 8.4 & $0-206.5$ \\
Hispanic & 7 & 12.6 & 2.4 & $0-47.6$ \\
African American & 7 & 4.2 & 0.8 & $0-14.1$ \\
Asian & 2 & 58.7 & 58.7 & $57.2-60.2$ \\
\hline
\end{tabular}

cancer after surgery for bone metastases. African Americans with prostate cancer survived for a significantly shorter time than did patients from other racial groups. Not surprisingly, disease progression was faster in African American men compared with others. Moreover, the shorter the time between diagnosis of skeletal metastasis and surgery for bone metastasis, the shorter the duration of cancer-pecific survival.

Our findings support a biological basis for the difference in outcome for African American men versus other races. Because the patient cohort of our study was from a single institution with similar criteria for treatment eligibility, the socioeconomic differences were minimal among ethnic groups. The shorter time period for disease progression in African American patients indicates a more aggressive disease in that group. Several studies have observed biological differences in prostate cancer between African American men and other ethnic groups; Castro et al. showed through genetic profiling that clinically localized cancers from African American men more closely resemble metastatic cancers from white men. Presently, there is no clear explanation for the more aggressive form of prostate cancer bone metastasis in African Americans. It was reported that African American men have higher bone density and/or higher androgen levels over their lifetime compared to those in other races [26]. More research is needed to clarify whether differences in the androgen levels or bone environment contribute to the accelerated progression of prostate cancer in bone.

Radiation treatment for bone metastasis, either before or after surgery, also was significantly associated with death from prostate cancer after surgery. Patients receiving radiation treatment tend to have more aggressive and advanced disease because radiation is typically given as a palliative treatment in hormone refractory cases. The Gleason score data before and after bone surgery differed for metastatic prostate cancer. The Gleason score, the most widespread method of prostate cancer tissue grading used today is the single most important prognostic factor in prostate cancer. The biopsy Gleason score is a sum of the primary grade (representing the majority of the tumor) and a secondary grade (assigned to the minority of the tumor), ranging from 2 to 10 . A higher Gleason score correlates with more aggressive disease, and a more unfavorable patient prognosis. Following prostate cancer diagnosis, a Gleason score of 9 or 10 was significantly associated with shorter survival (Figure 3); however, following bone metastasis surgery, this association did not reach statistical significance $(P=0.065)$. The Gleason score is based on the architectural pattern and degree of glandular differentiation of prostate cancer [21]. Cheville et al. found that the Gleason score of bone metastasis is associated with survival following surgery for metastasis, which supports the prognostic value of the Gleason score for men with prostate cancer undergoing surgery for bone metastases [7]. Our findings seem to be in agreement with this observation; we noted a trend for decrease in survival following surgery in men with high Gleason score prostate cancer, although this trend did not quite achieve statistical significance (HR, $2.58 ; P=0.065)$. A larger cohort may help to clarify the post-surgical risks.

We found that diagnosis of prostate cancer at an earlier age was independently associated with worse overall survival but had no effect on survival following surgery for bone metastases. There was no correlation between race and age of diagnosis that could be detected, due to the small sample size. This is consistent with other reports: Younger age at the time of diagnosis is thought to correlate with a more aggressive disease phenotype [27]. Higher PSA levels at time of diagnosis were associated with an increased risk of death, but not after surgery for prostate cancer bone metastases. Other studies have found evidence for and against an association of survival with PSA levels. In one of the few studies that focused on bone metastasis, Cheville et al. found no correlation between PSA levels at the time of bone metastasis diagnosis and survival after surgery, in agreement with our study [7]. PSA levels in their patient cohort (mean of 213, $\mathrm{n}=36$ ) were also similar to ours (mean of $187, \mathrm{n}=79$ ). A higher PSA level, as identified by our patient stratification, may indicate a more aggressive disease and thus a 
worse pre-surgical prognosis.

\subsection{Limitations}

This study has several limitations. The cohort size was small despite surveying 3.5 decades of patient records, which limitsared the power to detect significant differences and identify the factors that affect patient outcomes. The patient numbers were especially small for otherthan-White races/ethnicities, which especially limited generalizations about ethnic groups other than Whites. Moreover, the retrospective study design resulted in nonuniform patient treatment choices and incomplete information about patients' clinical characteristics. The numbers of patients in each racial group were dictated by chance rather than an even distribution, limiting the analysis among racial groups. Despite these limitations, our study has reinforced the need to look beyond demographics and access to care. We believe that the results of this study should be further investigated in a larger prospective group to clarify and further analyze the effect of immunobiologic tumor characteristics.

In summary, the results of the current study found that cancer-specific survival following surgery for prostate cancer bone metastasis is the poorest in African American men and in those treated with external radiation. Gleason score, age, and PSA levels can provide useful prognostic information following prostate cancer diagnosis prior to bone metastasis surgery.

\subsection{Conclusion}

Younger age at diagnosis (less than 65 years), African American ethniciity, higher PSA levels, higher Gleason scores, and radiation treatment all were associated with significantly worse survival outcomes following diagnosis of prostate cancer. Cancer-specific survival duration following surgery for prostate cancer bone metastasis is shortest in African American men. In this study, aggressive disease rather than lack of access to treatment appears more responsible for poor outcomes in African American men. Our study suggests that there are biological differences that underlie the difference in progression of prostate cancer in bone between African American men and other ethnic groups.

\section{Acknowledgements}

This work was supported by American Recovery and Reinvestment Act award number 5RC2MD004764-02 from the National Center on Minority Health and Health Disparities, National Institutes of Health, Bethesda, MD.

\section{REFERENCES}

[1] P. Abrahamsson, "Pathophysiology of Bone Metastases in Prostate Cancer," European Urology Supplements, Vol. 3, No. 5, 2004, pp. 3-9. doi:10.1016/j.eursup.2004.09.001

[2] R. E. Coleman, "Metastatic Bone Disease: Clinical Features, Pathophysiology and Treatment Strategies," Cancer Treatment Reviews, Vol. 27, No. 3, 2001, pp. 165-176. doi:10.1053/ctrv.2000.0210

[3] D. S. Ernst, J. Hanson and P. M. Venner, "Analysis of Prognostic Factors in Men with Metastatic Prostate Cancer. Uro-Oncology Group of Northern Alberta," Journal of Urology, Vol. 146, No. 2, 1991, pp. 372-376.

[4] M. J. Morris and H. I. Scher, "Clinical Approaches to Osseous Metastases in Prostate Cancer," Oncologist, Vol. 8, No. 2, 2003, pp. 161-173. doi:10.1634/theoncologist.8-2-161

[5] M. S. Soloway, S. W. Hardeman, D. Hickey, et al., "Stratification of Patients with Metastatic Prostate Cancer Based on Extent of Disease on Initial Bone Scan," Cancer, Vol. 61, No. 6, 1988, pp. 195-202. doi:10.1002/1097-0142(19880101)61:1<195::AID-CNCR 2820610133>3.0.CO;2-Y

[6] P. Castro, C. J. Creighton, M. Ozen, et al., "Genomic Profiling of Prostate Cancers from African American Men," Neoplasia, Vol. 11, No. 3, 2009, pp. 305-312.

[7] J. C. Cheville, D. Tindall, C. Boelter, et al., "Metastatic Prostate Carcinoma to Bone: Clinical and Pathologic Features Associated with Cancer-Specific Survival," Cancer, Vol. 95, No. 5, 2002, pp. 1028-1036. doi: $10.1002 /$ cncr. 10788

[8] R. M. Hoffman, F. D. Gilliland, J. W. Eley, et al., "Racial and Ethnic Differences in Advanced-Stage Prostate Cancer: The Prostate Cancer Outcomes Study," Journal of the National Cancer Institute, Vol. 93, No. 5, 2001, pp. 388395. doi:10.1093/jnci/93.5.388

[9] I. Thompson, C. Tangen, A. Tolcher, et al., "Association of African-American Ethnic Background with Survival in Men with Metastatic Prostate Cancer," Journal of the National Cancer Institute, Vol. 93, No. 3, 2001, pp. 219-225. doi:10.1093/jnci/93.3.219

[10] T. A. Wallace, R. L. Prueitt, M. Yi, et al., "Tumor Immunobiological Differences in Prostate Cancer between African-American and European-American Men," Cancer Research, 68, No. 3, 2008, pp. 927-936. doi:10.1158/0008-5472.CAN-07-2608

[11] R. A. Morton Jr., "Racial Differences in Adenocarcinoma of the Prostate in North American Men," Urology, Vol. 44, No. 5, 1994, pp. 637-645. doi:10.1016/S0090-4295(94)80196-7

[12] K. J. Pienta and P. S. Esper, "Risk Factors for Prostate Cancer," Annals of Internal Medicine, Vol. 118, No. 10, 1993, pp. 793-803. doi:10.7326/0003-4819-118-10-199305150-00007

[13] J. L. Stanford, R. A. Stephenson, L. M. Coyle, et al., "Prostate Cancer Trends 1973-1995," National Cancer Institute, Bethesda, 1999.

[14] C. M. Tangen, M. H. Hussain, C. S. Higano, et al., "Improved Overall Survival Trends of Men with Newly Diagnosed M1 Prostate Cancer: A SWOG Phase III Trial Experience (S8494, S8894 and S9346)," Journal of Uro- 
logy, Vol. 188, No. 4, 2012, pp. 1164-1169. doi:10.1016/j.juro.2012.06.046

[15] T. C. Brand, C. Bermejo, E. Canby-Hagino, et al., "Association of Polymorphisms in TGFB1 and Prostate Cancer Prognosis," Journal of Urology, Vol. 179, No. 2, 2008, pp. 754-758. doi:10.1016/j.juro.2007.09.020

[16] A. Berruti, L. Dogliotti, R. Bitossi, et al., "Incidence of Skeletal Complications in Patients with Bone Metastatic Prostate Cancer and Hormone Refractory Disease: Predictive Role of Bone Resorption and Formation Markers Evaluated at Baseline," Journal of Urology, Vol. 164, No. 4, 2000, pp. 1248-1253. doi:10.1016/S0022-5347(05)67149-2

[17] R. E. Coleman, "Skeletal Complications of Malignancy," Cancer, Vol. 80, Suppl. 8, 1997, pp. 1588-1594. doi:10.1002/(SICI)1097-0142(19971015)80:8+<1588::AI D-CNCR9>3.0.CO;2-G

[18] C. S. Galasko, "Skeletal Metastases," Clinical Orthopaedics and Related Research, Vol. 210, 1986, pp. 18-30.

[19] J. C. Bailar 3rd, G. T. Mellinger and D. F. Gleason, "Survival Rates of Patients with Prostatic Cancer, Tumor Stage, and Differentiation-Preliminary Report," Cancer Chemotherapy Reports, Vol. 50, No. 3, 1966, pp. 129136.

[20] D. F. Gleason, "Classification of Prostatic Carcinomas," Cancer Chemotherapy Reports, Vol. 50, No. 3, 1966, pp. 125-128.

[21] D. F. Gleason, "Histologic Grading and Clinical Staging of Prostatic Carcinoma," Lea \& Febiger, Philadelphia, 1977.

[22] D. F. Gleason, "Histologic Grading of Prostate Cancer: A Perspective," Human Pathology, Vol. 23, No. 3, 1992, pp. 273-279. doi:10.1016/0046-8177(92)90108-F

[23] D. F. Gleason and G. T. Mellinger, "Prediction of Prognosis for Prostatic Adenocarcinoma by Combined Histological Grading and Clinical Staging," Journal of Urology, Vol. 111, No. 1, 1974, pp. 58-64.

[24] K. K. Hodge, J. E. McNeal, M. K. Terris and T. A. Stamey, "Random Systematic versus Directed Ultrasound Guided Transrectal Core Biopsies of the Prostate," Journal of Urology, Vol. 142, No. 1, 1989, pp. 71-74; Discussion 4-5.

[25] E. Kaplan and P. Meier, "Nonparametric Estimation from Incomplete Observations," Journal of the American Statistical Association, Vol. 53, No. 282, 1958, pp. 457-481. doi:10.1080/01621459.1958.10501452

[26] A. K. Morgans, M. L. Hancock, K. G. Barnette, et al., "Racial Differences in Bone Mineral Density and Fractures in Men Receiving Androgen Deprivation Therapy for Prostate Cancer," Journal of Urology, Vol. 187, No. 3, 2012, pp. 889-893. doi:10.1016/j.juro.2011.10.136

[27] D. W. Lin, M. Porter and B. Montgomery, "Treatment and Survival Outcomes in Young Men Diagnosed with Prostate Cancer: A Population-Based Cohort Study," Cancer, Vol. 115, No. 13, 2009, pp. 2863-2871. doi: $10.1002 /$ cncr. 24324 